\title{
HEVnet: a One Health, collaborative, interdisciplinary network and sequence data repository for enhanced hepatitis E virus molecular typing, characterisation and epidemiological investigations
}

\author{
Annemieke Christine Mulder ${ }^{1}$, Annelies Kroneman ${ }^{1}$, Eelco Franz ${ }^{1}$, Harry Vennema ${ }^{1}$, Anna D. Tulen ${ }^{1}$, Johanna Takkinen ${ }^{2}$, Agnetha \\ Hofhuis ${ }^{1,3}$, Cornelia Adlhoch ${ }^{2,3}$, Members of HEVnet ${ }^{4}$ \\ 1. Centre for Infectious Disease Control, National Institute for Public Health and the Environment (RIVM), Bilthoven, the \\ Netherlands \\ 2. European Centre for Disease Prevention and Control (ECDC), Stockholm, Sweden \\ 3. These authors contributed equally to this article and share last authorship \\ 4. The members of HEVnet are listed at the end of the article
}

Correspondence: Agnetha Hofhuis (hevnet@rivm.nl)

Citation style for this article:

Mulder Annemieke Christine, Kroneman Annelies, Franz Eelco, Vennema Harry, Tulen Anna D., Takkinen Johanna, Hofhuis Agnetha, Adlhoch Cornelia, Members of HEVnet. HEVnet: a One Health, collaborative, interdisciplinary network and sequence data repository for enhanced hepatitis E virus molecular typing, characterisation and epidemiological investigations. Euro Surveill. 2019;24(10):pii=1800407. https://doi.org/10.2807/1560-7917.ES.2019.24.10.1800407

Hepatitis E virus (HEV) is a common cause of acute hepatitis worldwide. In Europe, HEV is a zoonosis transmitted via contaminated pork meat or other pork food products. Genotype 3 is the most prevalent HEV type in the animal reservoir, as well as in humans. Despite an increased incidence of hepatitis E across Europe, much remains unknown about its spread, sources and transmission routes. A One Health approach is crucial to better understand the (molecular) epidemiology of HEV. HEVnet was established in April 2017 as a network and database for sharing sequences and accompanying metadata collected from human, animal, food and environmental sources. HEVnet members working in the public health, veterinary health, food, environmental and blood safety sectors have submitted 1,615 HEV sequences from nine countries as at January 2019. Most are from humans ( $89 \%)$, and sequences of animal ( $5 \%)$, food $(6 \%)$ or environmental $(0.3 \%)$ origin are rare. Metadata for human sequences capture mostly sex (93\%), year of birth (92\%) and sampling (100\%); data on region of sampling (37\%) and clinical information (hospitalisation $27 \%$, symptoms $20 \%$ or mortality $8 \%$ ) are limited. HEVnet aims to expand into a global network capable of performing cross-sectoral and supranational studies, with a joint repository of molecular and epidemiological data on HEV.

\section{Introduction}

Hepatitis E virus (HEV) is one of the most common causative agents of acute hepatitis worldwide, with four major genotypes (1-4) affecting humans. Genotypes 1 and 2 are endemic in tropical and subtropical regions of Africa and Asia, where hepatitis $E$ is a waterborne disease associated with sporadic cases and large outbreaks via faecal-oral transmission or contaminated water. $\mathrm{HEV}_{1}$ and $\mathrm{HEV}_{2}$ infections in Europe and the United States (US) are travel associated [1]. In Europe, hepatitis E is considered a zoonosis transmitted via contaminated pork meat or other pork food products. Genotype 3 and, to a lesser degree, genotype 4 are prevalent in the animal reservoir (pigs, wild boar and deer), as well as in humans [2]. There are occasional reports of HEV transmission through other routes, such as shellfish, salad or vegetables contaminated by sewage water carrying HEV from infected animals or humans [2-7]. Infection through contaminated blood products or other substances of human origin have been reported, and some European countries (the United Kingdom, followed by Ireland and the Netherlands) have implemented comprehensive testing of blood donations to reduce infections among patients who are vulnerable to developing chronic hepatitis $E$ [8]. However, food appears to be the main transmission route in Europe.

A One Health approach is necessary to understand and mitigate HEV transmission via substances of human origin, animals, food and the environment [2]. Collaboration across disciplines is also crucial for the implementation of prevention and control measures that benefit public health, as HEV does not cause disease in animals and is therefore not considered a relevant veterinary issue requiring animal disease-control measures $[9,10]$.

According to hepatitis E-specific surveillance systems in 20 European Union (EU)/European Economic Area (EEA) countries, more than 20,000 cases were reported 
TABLE 1

Overview of data and experts required to reach the objectives of HEVnet

\begin{tabular}{|c|c|c|c|c|c|c|c|}
\hline \multicolumn{2}{|c|}{ Requirements } & $\begin{array}{l}\text { 1. Distribution } \\
\text { and trends } \\
\text { of circulating } \\
\text { human HEV } \\
\text { subtypes }^{\mathrm{a}}\end{array}$ & $\begin{array}{l}\text { 2. Molecular } \\
\text { epidemiology }\end{array}$ & $\begin{array}{l}\text { 3. Cluster } \\
\text { investigation }\end{array}$ & $\begin{array}{l}\text { R. } \\
\text { Relationship } \\
\text { of viruses } \\
\text { for source } \\
\text { attribution }\end{array}$ & $\begin{array}{l}\text { 5. Virus } \\
\text { evolution } \\
\text { and } \\
\text { spreade }\end{array}$ & $\begin{array}{l}\text { 6. Clinical } \\
\text { virology: } \\
\text { severity of } \\
\text { viral (sub) } \\
\text { types }^{f}\end{array}$ \\
\hline \multirow{6}{*}{ Data } & $\begin{array}{c}\text { Human origin sequences and } \\
\text { typing results according to } \\
\text { standardised nomenclature, } \\
\text { with information on time and } \\
\text { place }\end{array}$ & Y & Y & Y & Y & Y & Y \\
\hline & $\begin{array}{l}\text { Animal/food/environmental } \\
\text { origin sequences and } \\
\text { typing results according to } \\
\text { standardised nomenclature, } \\
\text { with time and place }\end{array}$ & $\mathrm{N}$ & $\mathrm{N}$ & $\mathrm{N}$ & $\mathrm{Y}$ & Y & $\mathrm{N}$ \\
\hline & $\begin{array}{l}\text { Sequences of an agreed } \\
\text { genomic region: minimum } \\
\text { length of } 300 \mathrm{nt} \text { within ORF2 }\end{array}$ & $\mathrm{N}$ & Y & Y & Y & Y & Y \\
\hline & $\begin{array}{c}\text { Standardised metadata: } \\
\text { demographic and } \\
\text { epidemiological }\end{array}$ & $\mathrm{N}$ & Y & $\mathrm{Y}$ & Y & N & $\mathrm{N}$ \\
\hline & $\begin{array}{c}\text { Standardised metadata: clinical } \\
\text { data }\end{array}$ & $\mathrm{N}$ & $\mathrm{N}$ & $\mathrm{N}$ & $\mathrm{N}$ & N & Y \\
\hline & Timely reporting & $\mathrm{N}$ & $\mathrm{N}$ & $Y$ & $\mathrm{Y}$ & $\mathrm{N}$ & $\mathrm{N}$ \\
\hline \multirow{4}{*}{ Expertise } & $\begin{array}{l}\text { Public health epidemiologists or } \\
\text { bioinformaticians }\end{array}$ & Y & Y & Y & Y & Y & Y \\
\hline & Clinical virologists & $\mathrm{N}$ & $Y$ & $Y$ & $\mathrm{Y}$ & $Y$ & $\mathrm{Y}$ \\
\hline & $\begin{array}{l}\text { Food/veterinary/environmental } \\
\text { virologists }\end{array}$ & $\mathrm{N}$ & $\mathrm{N}$ & $\mathrm{N}$ & Y & Y & $\mathrm{N}$ \\
\hline & Physicians & $\mathrm{N}$ & $\mathrm{N}$ & $Y$ & $\mathrm{~N}$ & $\mathrm{~N}$ & $Y$ \\
\hline
\end{tabular}

HEV: hepatitis E virus.

${ }^{a}$ Objective 1: to use molecular typing to assess the distribution and trends of HEV genotypes and subtypes circulating in humans.

${ }^{b}$ Objective 2: to analyse the distribution of subtypes in humans, leading to a better understanding of the underlying epidemiology of HEV.

c Objective 3: to combine human sequences with provided metadata of human cases, for cluster investigation.

${ }^{d}$ Objective 4: to trace the most likely reservoirs and sources of HEV by identifying connections between human and non-human (food, animal and environmental) samples via source attribution studies.

e Objective 5: to do population genetics studies into the spread and evolution of HEV.

${ }^{f}$ Objective 6: to assess the pathogenicity of strains through assessment of relationships between viral subtypes and severity of disease, looking at symptoms, hospitalisation and mortality.

between 2005-15, of which most were autochthonous $[8,11]$; more than $50 \%$ of the cases with known clinical information were reported from hospital settings and five countries reported a total of 28 fatal cases related to a HEV infection during this period [8]. Based on these reports, the European Centre for Disease Prevention and Control (ECDC) identified a need to support activities related to the investigation and assessment of HEV in the EU/EEA.

During the first ECDC HEV expert meeting in December 2015-attended by nominated scientists working on HEV-related topics in the EU/EEA, the World Health Organization (WHO) and the European Food Safety Agency (EFSA)-the development of a joint database of HEV sequences with metadata to support molecular epidemiological investigations was set as a priority, as molecular sequences provide more discriminatory power than only genotypes and subtypes of the virus $[8,12]$. The subsequent year, the ECDC and its HEV expert group further developed the concept of the database in collaboration with The National Institute for Public Health and the Environment (RIVM). RIVM already hosted pathogen-specific online databases such as HAVnet (for hepatitis A viruses) and NoroNet (for noroviruses), which are tools that have supported international investigations of outbreaks and molecular trends through standardisation and sharing of protocols [13-15].

This manuscript aims to introduce HEVnet (http://www. hevnet.nl), an international, cross-disciplinary database of HEV sequence data retrieved from different sources (humans, animals, the environment and food) and accompanied by relevant metadata for analysing the molecular epidemiological relationships between circulating HEV strains. 
TABLE 2

Overview of metadata reported for 1,615 sequences submitted to HEVnet, April 2017-January 2019

\begin{tabular}{|l|c|c|}
\hline Metadata with sequences & N & $\%$ \\
\hline Sample origin is reported & & $1,615)$ \\
\hline \multicolumn{1}{|c|}{ Human origin } & 1,443 & $89 \%$ \\
\hline \multicolumn{1}{|c|}{ Animal origin } & 75 & $5 \%$ \\
\hline \multicolumn{1}{|c|}{ Food origin } & 92 & $6 \%$ \\
\hline Year of sampling is reported & 5 & $0 \%$ \\
\hline Sequencing method is reported & 1,615 & $100 \%$ \\
\hline $\begin{array}{l}\text { Region of sampling is reported } \\
\text { (province or higher resolution) }\end{array}$ & 1,592 & $99 \%$ \\
\hline $\begin{array}{l}\text { Metadata with sequences of human } \\
\text { origin }\end{array}$ & 1,443 & $35 \%$ \\
\hline Sequencing method is reported & 1,420 & $98 \%$ \\
\hline Patient sex is reported & 1,349 & $93 \%$ \\
\hline Patient year of birth is reported & 1,332 & $92 \%$ \\
\hline Year of sampling is reported & 1,443 & $100 \%$ \\
\hline Hospitalisation ‘yes/no' is reported & 391 & $27 \%$ \\
\hline Symptoms are reported & 293 & $20 \%$ \\
\hline Mortality 'yes/no' reported & 109 & $8 \%$ \\
\hline $\begin{array}{l}\text { Region of sampling is reported } \\
\text { (province or higher resolution) }\end{array}$ & 528 & $37 \%$ \\
\hline
\end{tabular}

\section{The HEVnet database}

The HEVnet database is a password-protected online environment for sharing and analysing HEV sequence data accompanied by metadata. The metadata provide information on the specimen origin (country of sampling, date, type), sample origin (human, food, environmental or animal), patient (sex, year of birth, symptoms, hospitalisation, underlying conditions, etc.), behavioural exposure (food worker, animal contact, wastewater contact, other) and clinical parameters such as organ transplant or blood transfusion. All data are anonymised to ensure that the original source of the sample (e.g. an individual) cannot be traced, as this type of data is subject to the European General Data Protection Regulation [16]. Upon uploading, all sequences are automatically typed by a publicly available, curated, phylogenetic typing tool (https://www. rivm.nl/mpf/typingtool/hev) in order to secure standardised genotype and subtype assignment using the full-length sequences of reference viruses for HEV typing and subtyping proposed by Smith et al. in 2016 $[17,18]$, supplemented with representatives of tentative new subtypes.

Data can be queried, analysed and visualised as pie charts, geographical maps and phylogenetic trees, using a set of analysis modules within the protected online working space. Members can retrieve whole sequences or a queried selection of the sequences in FastA format for phylogenetic analyses. The metadata, with a link to the sequences, can be downloaded in Excel format for data analyses. The combination of phylogenetic and epidemiological data enables virological, epidemiological or combined investigations.

\section{HEVnet network}

The HEVnet network consists of HEV experts, such as virologists or epidemiologists in the public health, veterinary health, food, environmental and blood safety sectors. Participation in HEVnet is voluntary. A data confidentiality agreement grants membership and use of the database. Members are expected to respect a 'quid pro quo' principle in using the HEVnet data. The complete dataset is available to HEVnet members, and data ownership remains with the data provider. Compliant with the GDPR, and to promote research benefiting public health, all sequence data with a restricted set of associated metadata become publicly available at a time specified by the data provider, but no later than 18 months after submission.

\section{HEVnet objectives}

At the first HEVnet meeting in October 2017, 16 network members, RIVM's HEVnet team and representatives of ECDC and EFSA agreed that the objectives of HEVnet are to use molecular typing to assess trends in circulating HEV genotypes and subtypes in humans at an international level and to analyse the distribution of HEV subtypes in humans for better understanding of the epidemiology (e.g. characteristics) of the affected populations and the geographical relationships between viruses. Furthermore, HEVnet supports the investigation of clusters across countries. The pooled analysis of sequences across disciplines generates evidence about the relationships between isolates from human, animal, food and environmental specimens, and may help to identify sources of infection and transmission routes. Increased availability of sequence information should enable more in-depth virological analysis to study viral evolution. Linking of case-based patient data with molecular information could also support clinical studies on the pathogenicity and severity of viral types and subtypes, as well as the association of genotypes and clinical manifestations.

Table 1 outlines the objectives of HEVnet, as well as the data requirements and expertise needed to meet these objectives, such as standardisation of the generating and reporting of sequence data. As a first step, the HEVnet members agreed that a minimum sequence length of $300 \mathrm{nt}$ within ORF2 is required for robust analyses of HEV. Further, results relevant to risk assessments or clinicians will be communicated to the respective stakeholders, e.g. EFSA and ECDC.

\section{Current and future uses of the HEVnet database}

Since the system was launched in April 2017, 41 individual accounts have been created in HEVnet for experts from 15 countries: Austria, Belgium, Denmark, England 
and Wales, Estonia, France, Germany, Ireland, Italy, the Netherlands, Portugal, Spain, Sweden, Switzerland and the US. With the exception of the one member from the US, all current experts in the network are from Europe. However, HEVnet aims to expand this collaboration into a global network.

HEVnet has the potential to become an important resource for the scientific community working in the field of HEV. With the currently available sequences, patterns in circulating HEV subtypes can be analysed to identify and propose new reference viruses and to harmonise the classification and nomenclature of genotypes and subtypes across countries. Future work will include the standardisation of sequencing protocols, an update of the reference set with tentative new subtypes (focussed on genotype 3) and analyses of the submitted data.

Table 2 provides an overview of the metadata reported for 1,615 sequences submitted by 15 institutes in nine countries for the period April 2017-January 2019. These sequences mainly originate from human cases (89\%), and are rarely of animal (5\%), food $(6 \%)$ or environmental origin (0.3\%). The number of sequences obtained from animals, food and the environment is not yet sufficient to investigate the HEV relationships across these disciplines, which is crucial for understanding the epidemiology of HEV. HEVnet encourages HEV experts from the veterinary, food and environmental sectors to join the network and support its growth by disseminating this invitation and connecting with projects of One Health initiatives, such as the One Health European Joint Programmes (https:// onehealthejp.eu/) and the Med-Vet-Net Association (http://mvnassociation.org/medvetnet/index.php).

Molecular epidemiological analyses focussing on human clinical data, including sequences from blood donors and patients with chronic HEV infection, might help to identify and better understand relationships between viruses, with regard to severity and associated risk factors. HEVnet allows collecting an array of clinically relevant parameters, such as duration and nature of symptoms, hospitalisation, comorbidity and treatment outcomes. However, as shown in Table 2, the majority of submitted sequences of human origin contain only metadata on patient sex (93\%), year of birth (92\%) and year of sampling (100\%). Far fewer sequences have metadata on the region of sampling $(37 \%)$ or clinical information such as hospitalisation $(27 \%)$, symptoms $(20 \%)$ or mortality $(8 \%)$. Improving the completeness of metadata in HEVnet could be accomplished if future studies link more variables of metadata to submitted sequences. For example, the Netherlands included a broad range of anonymised metadata when they submitted all available HEV sequences from a recent nationwide patient control study into risk factors of HEV [19]. Authors of future studies in which HEV sequences are collected together with metadata are encouraged to connect with HEVnet so they can benefit from the network collaboration by comparing their newly collected sequences to the worldwide repository. The availability of large datasets and full-length sequences will enable the refinement of current subgenotype classification and the identification of new divergent subtypes in both humans and animals, over time.

\section{Conclusion}

Using a joint repository of molecular and epidemiological data, HEVnet enables cross-sectoral and supranational collaborations to conduct molecular epidemiological investigations on HEV. A One Health approach is necessary to understand and mitigate HEV transmission via food, animals, substances of human origin and the environment. To strengthen the HEVnet database as an international One Health platform, it is necessary that more experts in the veterinary, food and environmental sectors join the network and submit their sequences with metadata. HEV experts across the world are invited to join the network by sending an email request (hevnet@rivm.nl) with a short explanation of what they would like to contribute to the network and the establishment of a worldwide reference set of HEV sequences.

\section{Members of HEVnet}

Stephan Aberle, Medical University of Vienna, Vienna, Austria

Lorenzo Subissi, Sciensano, Brussels, Belgium

Vanessa Suin, Sciensano, Brussels, Belgium

Sofie Midgley, Virus surveillance and research, Statens Serum Institut, Copenhagen, Denmark

Tatiana Kuznetsova, Department of Virology and Immunology, National Institute for Health Development, Tallinn, Estonia

Jacques Izopet, Federative Intitute of Biology, National Reference Center for Hepatitis E virus, Toulouse, France

Nicole Pavio, French Agency for Food, Environmental and Occupational Health and Safety (ANSES), Maisons-Alfort, France

Christine Baechlein, University of Veterinary Medicine Hannover, Hannover, Germany

Sally A. Baylis ${ }^{\text {a }}$ Paul-Ehrlich-Institut (PEI), Langen, Germany

Victor M. Corman, Institute of Virology Charité Universitätsmedizin Berlin, Berlin, Germany

Mirko Faber ${ }^{a}$, Robert Koch-Institut, Berlin, Germany

Reimar Johne, German Federal Institute for Risk Assessment (BfR), Berlin, Germany

Christel Kamp, Paul-Ehrlich-Institut (PEI), Langen, Germany

Juergen J. Wenzel, Institute of Clinical Microbiology and Hygiene, Regensburg University Medical Center, Regensburg, Germany 
Suzie Coughlan, National virus reference laboratory, University College Dublin, Dublin, Ireland

Ilaria Di Bartolo, Istituto Superiore di Sanità, Rome, Italy

Roberto Bruni, Istituto Superiore Di Sanità, Rome, Italy

Anna Rita Ciccaglionea ${ }^{a}$ Istituto Superiore di Sanità, Rome, Italy

Anna Rosa Garbuglia, Laboratory of virology INMI Spallanzani IRCCs Italy

Elisabetta Suffredini, Department of Food safety, Nutrition and Veterinary public health, Istituto Superiore di Sanità, Rome, Italy

Ingeborg Boxman, the Netherlands Food and Consumer Product Safety Authority (NVWA), the Netherlands

Boris Hogema, Sanquin Blood Supply Foundation, Amsterdam, the Netherlands

Wim van der Poel, Wageningen BioVeterinary Research, Wageningen, the Netherlands

Hans Zaaijera, Sanquin Blood Supply Foundation, Amsterdam, the Netherlands

Rita de Sousa a Instituto Nacional de Saúde Doutor Ricardo Jorge, Lisbon, Portugal

Branko Velebit, Institute of Meat Hygiene and Technology, Serbia

Ana Avellón ${ }^{a}$, Viral Hepatitis Reference and Research Laboratory National Center of Microbiology Carlos III Health Institute, Madrid, Spain

Maria Buti, Hospital Universitario Valle Hebron, Barcelona, Spain

Rosina Girones, Section of Microbiology, Virology and Biotechnology Department of Genetics, Microbiology and Statistics Faculty of Biology University of Barcelona, Barcelona, Spain

Josep Quer, Vall d'Hebron Institut Research, Barcelona, Spain

Frederik Widén, National Veterinary Institute (SVA), Uppsala, Sweden

Heléne Norder, Sahlgrenska University Hospital, Gothenburg, Sweden

Kristina Nyström, Sahlgrenska University Hospital, Gothenburg, Sweden

Claudia Bachofen, Institute of Virology, Vetsuisse Faculty of the University of Zürich, Zürich, Switzerland

Roland Sahli, Institute of microbiology, Centre Hospitalier Universitaire Vaudois and University of Lausanne, Lausanne, Switzerland

Harry Daltona, Royal Cornwall Hospital, Treliske, UK

Samreen Ijaza, Public Health England, London, UK

Samantha Treagus, CEFAS, University of Exeter, UK

Michael Kulka, Center for Food Safety and Applied Nutrition, U.S. Food and Drug Administration, Unites States of America
Valentina Rizzi, European Food Safety Authority (EFSA), Unit of Biological Hazards and Contaminants, Parma, Italy

a Also a member of the European Centre for Disease Prevention and Control (ECDC) HEV expert group.

\section{Acknowledgements}

We thank all people involved during the development of HEVnet for their support.

Disclaimer: The views and opinions expressed herein are the authors' own and do not necessarily state or reflect those of the European Centre for Disease Prevention and Control $(E C D C)$. ECDC is not responsible for the data or the information collation and analysis.

The positions and opinions presented in this article are those of the authors alone and are not intended to represent the views or scientific works of the European Food Safety Agency (EFSA).

Funding statement: The National Institute for Public Health and the Environment (RIVM) developed and maintains the HEVnet database, financed by the Ministry of Health, Welfare and Sport of the Netherlands. The funding ministry had no role in the design of HEVnet, data collection, decision to publish or preparation of this manuscript. The development of accompanying documents, e.g. confidentiality agreement, was supported by ECDC internal funds (contract no. NP/2016/OCS/7892/01). All documents related to the ECDC contract and funding of the meetings, as well as review of this manuscript, have been subject to formal ECDC governance procedures. ECDC has no access to the HEVnet database and therefore plays no role in the data collection, analysis or other technical set-up related to the database or the network. ECDC and RIVM funded the first HEVnet meeting at the RIVM in October 2017.

\section{Conflict of interest}

None declared.

\section{Authors' contributions}

Manuscript conceptualization, investigation, methodology and resources: $A C M, A H, A K, H V, E F, C A$ and the HEVnet members. Data curation, software, analyses: ACM, AK and HV. Funding acquisition: CA and EF. Project administration: ACM, AK, CA, EF and HV. Supervision: AH, AK and EF. Visualization: $A C M, A H$ and CA. Writing - original draft preparation: ACM. Writing - review and editing: ACM, AH, CA, EF, JT, AT and the HEVnet members.

\section{References}

1. Kamar N, Izopet J, Pavio N, Aggarwal R, Labrique A, Wedemeyer $\mathrm{H}$, et al. Hepatitis E virus infection. Nat Rev Dis Primers. 2017;3:17086. https://doi.org/10.1038/nrdp.2017.86 PMID: 29154369

2. Ricci A, Allende A, Bolton D, Chemaly M, Davies R, Fernandez Escamez PS, et al. Public health risks associated with hepatitis E virus (HEV) as a food-borne pathogen. EFSA J. 2017;15(7)4886.

3. Colson P, Borentain P, Queyriaux B, Kaba M, Moal V, Gallian $P$, et al. Pig liver sausage as a source of hepatitis $E$ virus transmission to humans. J Infect Dis. 2010;202(6):825-34. https://doi.org/10.1086/655898 PMID: 20695796

4. Hawkes N. Pork is UK's main source of hepatitis E infection, briefing hears. BMJ. 2014:349(nov11 11):g6779. https://doi. org/10.1136/bmj.g6779 PMID: 25391551 
5. Riveiro-Barciela M, Mínguez B, Gironés R, Rodriguez-Frías F, Quer J, Buti M. Phylogenetic demonstration of hepatitis E infection transmitted by pork meat ingestion. J Clin Gastroenterol. 2015;49(2):165-8. https://doi.org/10.1097/ MCG.0000000000000113 PMID: 24637729

6. Said B, ljaz S, Chand MA, Kafatos G, Tedder R, Morgan D. Hepatitis E virus in England and Wales: indigenous infection is associated with the consumption of processed pork products. Epidemiol Infect. 2014;142(7):1467-75. https://doi.org/10.1017/ So950268813002318 PMID: 24054519

7. Said B, ljaz S, Kafatos G, Booth L, Thomas HL, Walsh A, et al. Hepatitis E outbreak on cruise ship. Emerg Infect Dis. 2009;15(11):1738-44. https://doi.org/10.3201/eid1511.091094 PMID: 19891860

8. European Centre for Disease Prevention and Control (ECDC). Hepatitis E in the EU/EEA, 2005-2015. Stockholm: ECDC; 2017. Available from: https://ecdc.europa.eu/en/publications-data/ hepatitis-e-eueea-2005-2015

9. Pavio N, Doceul V, Bagdassarian E, Johne R. Recent knowledge on hepatitis E virus in Suidae reservoirs and transmission routes to human. Vet Res (Faisalabad). 2017;48(1):78. https:// doi.org/10.1186/s13567-017-0483-9 PMID: 29157309

10. Van der Poel WHM, Dalton HR, Johne R, Pavio N, Bouwknegt $\mathrm{M}$, Wu T, et al. Knowledge gaps and research priorities in the prevention and control of hepatitis E virus infection. Transbound Emerg Dis. 2018;65(Suppl 1):22-9. https://doi. org/10.1111/tbed.12760 PMID: 29318757

11. Aspinall EJ, Couturier E, Faber M, Said B, ljaz S, Tavoschi L, et al. Hepatitis E virus infection in Europe: surveillance and descriptive epidemiology of confirmed cases, 2005 to 2015. Euro Surveill. 2017;22(26):30561. https://doi.org/10.1016/j. jcv.2016.06.010 PMID: 27393938

12. Adlhoch C, Avellon A, Baylis SA, Ciccaglione AR, Couturier E, de Sousa R, et al. Hepatitis E virus: Assessment of the epidemiological situation in humans in Europe, 2014/15. J Clin Virol. 2016;82:9-16. https://doi.org/10.1016/j.jcv.2016.06.010 PMID: 27393938

13. Kroneman A, de Sousa R, Verhoef L, Koopmans MPG, Vennema HOn Behalf Of The HAVNet Network. Usability of the international HAVNet hepatitis A virus database for geographical annotation, backtracing and outbreak detection. Euro Surveill. 2018;23(37):1700802. https:// doi.org/10.2807/1560-7917.ES.2018.23.37.1700802 PMID: 30229723

14. Niesters HG, Rossen JW, van der Avoort H, Baas D, Benschop K, Claas EC, et al. Laboratory-based surveillance in the molecular era: the TYPENED model, a joint data-sharing platform for clinical and public health laboratories. Euro Surveill. 2013;18(4):20387. https://doi.org/10.2807/ese.18.04.20387en PMID: 23369392

15. van Beek J, de Graaf M, Al-Hello H, Allen DJ, Ambert-Balay $\mathrm{K}$, Botteldoorn $\mathrm{N}$, et al. Molecular surveillance of norovirus, 2005-16: an epidemiological analysis of data collected from the NoroNet network. Lancet Infect Dis. 2018;18(5):54553. https://doi.org/10.1016/S1473-3099(18)30059-8 PMID: 29396001

16. European Commission. Commission Regulation (EC) No $2016 / 679$ of 27 April 2016 on the protection of natural persons with regard to the processing of personal data and on the free movement of such data, and repealing Directive 95/46/ EC (General Data Protection Regulation). Official Journal of the European Union. Luxembourg: Publications Office of the European Union. 04.05.2016:L 119. Available from: https://eurlex.europa.eu/legal-content/EN/TXT/?uri=0J\%3AL\%3A2016\%3 A $119 \% 3$ ATOC

17. Smith DB, Simmonds P, Izopet J, Oliveira-Filho EF, Ulrich RG Johne R, et al. Proposed reference sequences for hepatitis $E$ virus subtypes. J Gen Virol. 2016;97(3):537-42. https://doi. org/10.1099/jgv.0.000393 PMID: 26743685

18. Kroneman A, Vennema H, Deforche K, v d Avoort H, Peñaranda $\mathrm{S}$, Oberste MS, et al. An automated genotyping tool for enteroviruses and noroviruses. J Clin Virol. 2011;51(2):121-5. https://doi.org/10.1016/j.jcv.2011.03.006 PMID: 21514213

19. Tulen AD, Vennema H, van Pelt W, Franz E, Hofhuis A. A casecontrol study into risk factors for acute hepatitis $E$ in the Netherlands, 2015-2017. J Infect. 2019;S0163-4453(19)30036-2. https://doi.org/10.1016/j.jinf.2019.02.001 PMID: 30738918 credit to the source, provide a link to the licence and indicate if changes were made.

Any supplementary material referenced in the article can be found in the online version.

This article is copyright of the authors or their affiliated institutions, 2019.

\section{License, supplementary material and copyright}

This is an open-access article distributed under the terms of the Creative Commons Attribution (CC BY 4.0) Licence. You may share and adapt the material, but must give appropriate 